\title{
Use of Core-Needle Biopsy for the Diagnosis of Malignant Lymphomas in Clinical Practice
}

\author{
Yuta Ito $^{a}$ Akiko Miyagi Maeshimab Shunsuke Hatta ${ }^{a}$ Yo Saito ${ }^{a}$ \\ Takahiro Fujino $^{a}$ Shinichi Makita ${ }^{a}$ Suguru Fukuhara ${ }^{a}$ Wataru Munakata ${ }^{a}$ \\ Hirokazu Taniguchia ${ }^{a}$ Tatsuya Suzuki $^{a}$ Dai Maruyama ${ }^{a}$ Miyuki Sone ${ }^{c}$ \\ Koji Izutsu ${ }^{a}$
}

aDepartment of Hematology, National Cancer Center Hospital, Tokyo, Japan; 'bepartment of Pathology, National Cancer Center Hospital, Tokyo, Japan; 'Department of Diagnostic Radiology National Cancer Center Hospital, Tokyo, Japan

\author{
Keywords \\ Core-needle biopsy · Excisional biopsy · Malignant \\ lymphoma $\cdot$ Diagnostic biopsy
}

\begin{abstract}
Introduction: Excisional biopsy (EB) is considered the gold standard for lymphoma diagnosis. Although recent advances in interventional radiology enable sampling with coreneedle biopsy (CNB), only few studies evaluated the utility of CNB compared to that of EB. Methods: We analyzed patients with lymphoma who had a diagnostic biopsy at the National Cancer Center Hospital during 2002-2017. We investigated the clinical and pathological characteristics of CNB in 2017. Results: The proportion of CNB utility in total biopsy procedures had increased from 11 to $48 \%$ during the 15 years. In 2017, CNB was opted more frequently than EB for a biopsy of superficial, abdominal, or anterior mediastinal lesions. Only one out of 72 patients who had CNB required re-biopsy with $E B$ because of insufficiency. The incidence of complications was comparable between CNB and EB: 2 (4\%) cases of minor bleeding with CNB and 1 (8\%) case of minor bleeding with $\mathrm{EB}$. The median time from the first visit to biopsy was significantly shorter with CNB (5.5 days) than with EB (15
\end{abstract}

karger@karger.com www.karger.com/aha

Karger"
(C) 2021 The Author(s)

Published by S. Karger AG, Base

This is an Open Access article licensed under the Creative Commons Attribution-NonCommercial-4.0 International License (CC BY-NC) (http://www.karger.com/Services/OpenAccessLicense), applicable to the online version of the article only. Usage and distribution for commercial purposes requires written permission. days). Conclusion: There is an increasing trend in the utility of CNB. CNB is a less invasive method with shorter time to biopsy and can be considered an alternative to EB.

(c) 2021 The Author(s).

Published by S. Karger AG, Basel

\section{Introduction}

Traditionally, excisional biopsy (EB) has been considered the gold standard method for the diagnosis of malignant lymphomas [1]. This is partly because evaluation of the whole histological architecture of the lymph nodes is important to distinguish lymphoma from other conditions including reactive lymphadenopathy or to determine the histological subtype of lymphoma. Furthermore, EB enables to obtain sufficient tissue samples for various ancillary tests including flow cytometry, cytogenetic analysis with fluorescence in situ hybridization (FISH), and molecular genetic tests. Currently, the World Health Organization (WHO) diagnostic schemes of malignant lymphomas and major clinical practice guidelines recommend EB as the primary method for lymphoma diagnosis [2-9]. EB might be a reasonable option for patients with superficial lymphadenopathy because superfi-
Correspondence to:

Koji Izutsu, kizutsu@ncc.go.jp 
cial lymph nodes are easy to access and the specimen can be obtained with outpatient surgery under local anesthesia. However, EB appears to be a more invasive procedure to obtain a specimen such as anterior mediastinal mass or abdominal lymph nodes from so-called "deep-seated lesions" because inpatient surgery under full anesthesia is essential. EB results in longer time to recovery and a higher risk of complications such as bleeding or infection than core-needle biopsy (CNB). Recent progress in interventional radiology (IR) has enabled obtaining a diagnostic specimen from any site, with some exceptions, using $\mathrm{CNB}$, which is a less invasive biopsy method. In recent decades, CNB has virtually become one of the initial diagnostic methods for lymphoma in many centers [10]. Additionally, the recently published literature review article confirmed the usefulness of CNB because of its diagnostic efficacy, reproducibility, and safety [11]. In this review, CNB demonstrated a high diagnostic rate (79-97\%), high reproducibility among pathologists (87-93\%), and minimal invasiveness. Therefore, the authors concluded that CNB is considered to be a first-line diagnostic approach in clinical practice.

However, there are only few studies evaluating the trend in the use of $\mathrm{CNB}$ and the characteristics of $\mathrm{CNB}$ when compared to EB as a diagnostic procedure for lymphoma. Therefore, our study aimed to assess the trend of utility and clinical and pathological characteristics of CNB for lymphoma diagnosis in clinical practice at our institution over the last 15 years.

\section{Materials and Methods}

\section{Patients and Outcomes}

In this retrospective study, to evaluate the trend of the frequency of CNB and EB in patients who were diagnosed with malignant lymphoma, we analyzed consecutive $73,78,87$, and 151 patients who underwent the primary diagnostic biopsy, regardless of the biopsy sites and were diagnosed as lymphoma at the National Cancer Center Hospital in 2002, 2007, 2012, and 2017. Based on the medical records, we classified the biopsy procedures into 4 groups as follows: CNB, EB, endoscopic biopsy, and others including, endobronchial ultrasound transbronchial needle aspiration, surgery except for lymph node biopsy, bone marrow biopsy, and centesis of body cavity fluid. For 151 patients who underwent diagnostic biopsy in 2017, we described the breakdown of the biopsy method. Moreover, to describe the characteristics of CNB in comparison with $\mathrm{EB}$ in an unbiased manner, we focused on 60 patients who underwent superficial, abdominal, or anterior mediastinal lymphadenopathy and were potentially subjected to either CNB or EB. We then described the distribution of the lesion, lesion size, and use of ancillary examinations including flow cytometry, conventional karyotyping, and FISH. We also explored the time from the first visit to biopsy and diagnosis. The endpoint of the biopsy pro- cedure was whether the clinicians can determine the treatment strategy based on the pathological results, the so-called actionable diagnosis [12]. The present study was approved by the Ethics Committee of the National Cancer Center (Approval number: 2017-161). Informed consent was waived because of the retrospective nature of the study.

\section{Biopsy Procedures}

In terms of $\mathrm{CNB}$, all procedures were performed under computed tomography or ultrasound guidance by expert physicians. An 18-gauge semiautomatic core needle was used in almost all the cases with at least 3 passes for each biopsy under a local anesthesia. As needed, some of them were analyzed by flow cytometry and Gbanding or FISH and/or cryopreserved for additional future examinations. Indication for these ancillary tests using fresh sample was decided at the discretion of hematologists who ordered the biopsy. Complications were described based on the Clavien-Dindo classification of the Japan Clinical Oncology Group [13].

\section{Pathologic Diagnosis}

All tissues for histology were fixed in 10\% neutral buffered formalin, embedded in paraffin, cut into 4 - $\mu$ m-thick sections, and stained with hematoxylin and eosin for histological evaluation. All specimens were diagnosed and further evaluated by immunohistochemistry and other ancillary studies including FISH on formalin-fixed paraffin-embedded tissue, as required. The diagnosis was made according to the latest lymphoma classification system available at the time of diagnosis.

\section{Statistical Analysis}

Categorical variables in the 2 groups were compared by Fisher's exact test. Continuous variables such as age, lesion size, number of slides for immunohistochemical assessment, time from the first visit to biopsy, and diagnosis were compared using the MannWhitney $\mathrm{U}$ test. All tests were 2 -sided, and a $p$ value $<0.05$ was considered statistically significant. All calculations were performed using EZR (Saitama Medical Center, Jichi Medical University, Saitama, Japan), a graphical user interface for R 2.13.0 (R Foundation for Statistical Computing, Vienna, Austria). More precisely, EZR is a modified version of R commander (version 1.63) designed to add statistical functions frequently used in biostatistics [14].

\section{Results}

\section{Trend in Increased Frequency of CNB from 2002 to} 2017

Out of $73,78,87$, and 151 patients who underwent primary diagnostic biopsy regardless of biopsy sites and were diagnosed with lymphoma in $2002,2007,2012$, and 2017 , respectively, the trend in the frequency of CNB and EB was analyzed. While the proportion of EB utility decreased from 43 to $9 \%$, the proportion of CNB utility significantly increased from $11 \%$ in 2002 to $48 \%$ in 2017 , as shown in Figure 1. 
Fig. 1. Transition of the frequency of CNB and EB over the 15 years from 2002 to 2017. $\mathrm{EB}$, excisional biopsy; CNB, core-needle biopsy.

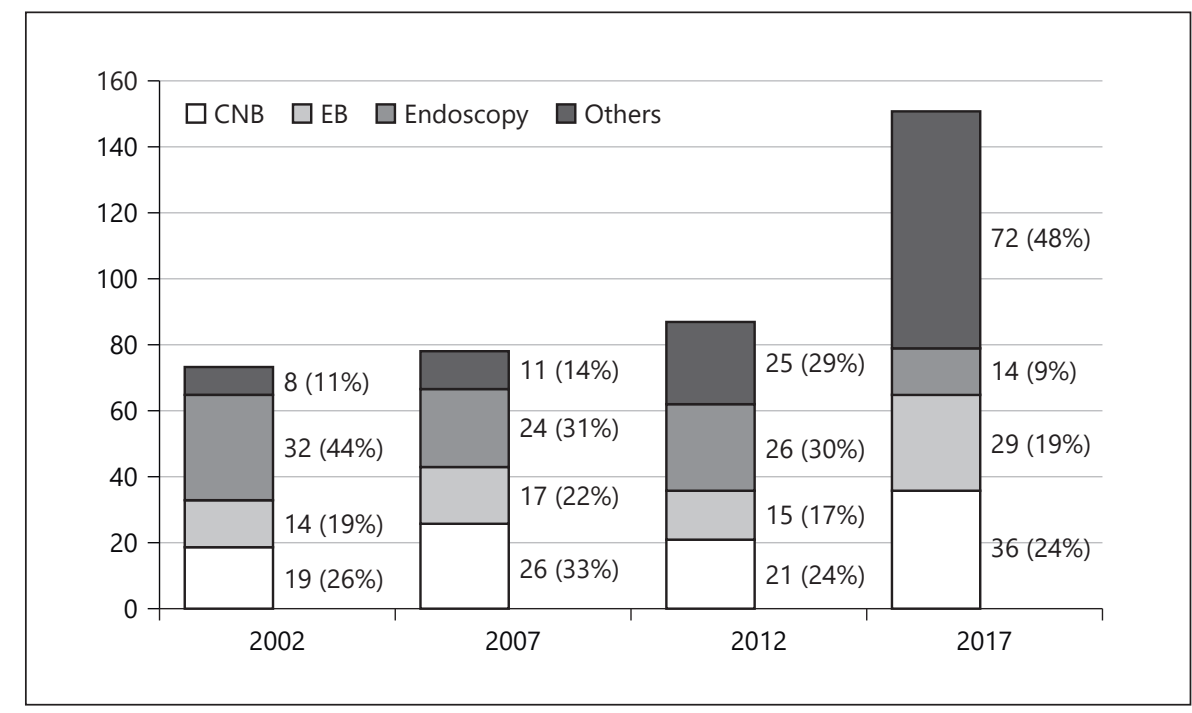

\section{Patients' Characteristics and Proportion of Each}

Biopsy Method in 2017

For the 151 patients who received diagnostic biopsy at our institution and were diagnosed with malignant lymphoma, the distribution of the histologic subtype is summarized in Table 1. There were 4 cases with low-grade B-cell lymphoma which could not be subclassified according to the World Health Organization (WHO) classification in the CNB cohort. In those 4 cases, follicular lymphoma, chronic lymphocytic leukemia/small lymphocytic leukemia, or mantle cell lymphoma was ruled out based on immunostaining studies (CD5, CD10, BCL2, and cyclin D1) and FISH (IGH/BCL2). The median patient age was 64 (range 18-89) years, and 87 patients (57.6\%) were male. Regarding biopsy methods other than CNB and EB, endoscopy was the second most frequent procedure at 19\% (29 patients). The remaining procedures including punch biopsy, endobronchial ultrasound transbronchial needle aspiration, surgery except for lymph node biopsy, bone marrow biopsy, and centesis of body cavity fluid accounted for $24 \%$ cases (36 patients).

\section{Summary of CNB Procedures}

In total, among 72 patients who underwent $\mathrm{CNB}$ in 2017 , an 18-gauge core needle was utilized most frequently at $67 \%$ (48 patients), followed by a 20 -gauge and a 13 -gauge core needle, which were used at $4 \%$ (3 patients) each. Information about the gauge of core needle was unavailable from medical records for the remaining 18 cases. The most frequent number of passes was 3 (range, 1 to 10 passes) in $49 \%$ ( 35 patients), followed by 4 in 19\%, 2 in
Table 1. Distribution of histologic subtypes

\begin{tabular}{lrr}
\hline Histology & $N$ & $\%$ \\
\hline Total & 151 & \\
B-cell neoplasms & 2 & 1.3 \\
$\quad$ CLL/SLL & 61 & 40.3 \\
DLBCL & 40 & 26.5 \\
FL & 2 & 1.3 \\
High-grade B-cell lymphoma & 4 & 2.6 \\
Low-grade B-cell lymphoma ${ }^{1}$ & 1 & 0.7 \\
LPL & 11 & 7.3 \\
MALT & 4 & 2.6 \\
MCL & 2 & 1.3 \\
Plasmablastic lymphoma & 1 & 0.7 \\
Nodal MZL & & \\
NK/T-cell neoplasms & 3 & 2.0 \\
$\quad$ AITL & 2 & 1.3 \\
ALCL & 3 & 1.3 \\
ATL & 1 & 0.7 \\
CTCL & 5 & 3.3 \\
ENKL & 9 & 6.0 \\
Hodgkin & & \\
\hline
\end{tabular}

AITL, angioimmunoblastic T-cell lymphoma; ALCL, anaplastic large cell lymphoma; ATL, adult T-cell leukemia/lymphoma; CTCL, cutaneous T-celllymphoma;CLL/SLL, chroniclymphocytic leukemia/small lymphocytic lymphoma; DLBCL, diffuse large B-cell lymphoma; ENKL, extranodal NK/T-cell lymphoma, nasal type; FL, follicularlymphoma; LPL, lymphoplasmacyticlymphoma; MALT, mucosa-associated lymphoid tissue lymphoma; MCL, mantle cell lymphoma; MZL, marginal zone lymphoma; FISH, fluorescence in situ hybridization.

${ }^{1}$ Despite full immunohistochemical studies including CD5, CD10, BCL2, and CyclinD1 and FISH studies, these 4 cases could not be subclassified. 
Table 2. Patient characteristics, use of ancillary tests, and days from first visit to biopsy of those having CNB or EB from anterior mediastinal lesion or superficial and intra-abdominal lymphadenopathy

\begin{tabular}{|c|c|c|c|}
\hline & $\begin{array}{l}\mathrm{CNB} \\
(n=47)(\%)\end{array}$ & $\begin{array}{l}\text { EB } \\
(n=13)(\%)\end{array}$ & $p$ value \\
\hline Age, age, median (range) & $67.00(26.00,89.00)$ & $61.00(18.00,80.00)$ & 0.66 \\
\hline \multicolumn{4}{|l|}{ Sex } \\
\hline Male & $27(57.4)$ & $7(53.8)$ & \multirow[b]{2}{*}{1} \\
\hline Female & $20(42.6)$ & $6(46.2)$ & \\
\hline \multicolumn{4}{|l|}{ Subtype } \\
\hline Aggressive $^{1}$ & $27(57.4)$ & $8(61.5)$ & \multirow[b]{2}{*}{1} \\
\hline Indolent ${ }^{2}$ & $20(42.6)$ & $5(38.5)$ & \\
\hline \multicolumn{4}{|l|}{ Histology } \\
\hline DLBCL & $17(36.2)$ & $3(23.0)$ & \\
\hline \multicolumn{4}{|l|}{ Cell of origin (only DLBCL) } \\
\hline GCB & 9 & 0 & \\
\hline Non-GCB & 6 & 2 & \\
\hline Unclassified & 2 & 1 & \\
\hline FL & $19(40.4)$ & $4(30.8)$ & \\
\hline Other B-cell lymphomas & $2(4.3)$ & $1(7.7)$ & \\
\hline T/NK-cell lymphoma & $4(8.5)$ & $2(15.4)$ & \\
\hline Hodgkin lymphoma & $5(10.6)$ & $3(23.1)$ & \\
\hline \multicolumn{4}{|l|}{ Distribution $^{3}$} \\
\hline Superficial & $22(46.8)$ & $12(92.3)$ & \multirow{2}{*}{0.004} \\
\hline Deep & $25(53.2)$ & $1(7.7)$ & \\
\hline \multicolumn{4}{|l|}{ Complication } \\
\hline Minor bleeding & $2(4.3)$ & $1(7.7)$ & \multirow[t]{2}{*}{0.526} \\
\hline None & 45 (95.7) & $12(92.3)$ & \\
\hline Nodal size, $\mathrm{cm}$ median (range) & $4.20(1.00,15.00)$ & $2.80(0.70,4.80)$ & 0.002 \\
\hline \multicolumn{4}{|l|}{ Number of immunohistochemistry } \\
\hline Slides, median (range) & $6(4,14)$ & $7(6,14)$ & 0.12 \\
\hline \multicolumn{4}{|l|}{ Ancillary tests } \\
\hline Flow cytometry & & & \\
\hline Yes & $4(8.5)$ & $10(76.9)$ & \multirow{2}{*}{$<0.001$} \\
\hline No & $43(91.5)$ & $3(23.1)$ & \\
\hline \multicolumn{4}{|l|}{ G-banding } \\
\hline Yes & $4(8.5)$ & $7(53.8)$ & \multirow{2}{*}{$<0.001$} \\
\hline No & $43(91.5)$ & $6(46.2)$ & \\
\hline \multicolumn{4}{|l|}{ FISH } \\
\hline Yes & $4(8.5)$ & 0 & \multirow{2}{*}{0.57} \\
\hline No & $43(91.5)$ & $13(100)$ & \\
\hline \multicolumn{4}{|l|}{ Days from the first visit to biopsy } \\
\hline Median (range) & $5.5(0.0,185.0)$ & $15.0(0.0,67.0)$ & 0.01 \\
\hline \multicolumn{4}{|l|}{ Days from the first visit to diagnosis } \\
\hline Median (range) & $15.0(6.0,192.0)$ & $22.0(12.0,80.0)$ & 0.004 \\
\hline
\end{tabular}

DLBCL, diffuse large B-cell lymphoma; GCB, germinal center B-cell; FL, follicular lymphoma; EB, excisional biopsy; CNB, core-needle biopsy; FISH, fluorescence in situ hybridization.

${ }^{1}$ Aggressive lymphoma included DLBCL, T/NK-cell lymphoma, Hodgkin lymphoma, and plasmablastic lymphoma.

${ }^{2}$ Indolent lymphoma included FL and low-grade B-cell lymphoma.

${ }^{3}$ Superficial lymphadenopathy includes cervical, axillary, or inguinal lesions. Deep lymphadenopathy includes anterior mediastinal and abdominal lymphadenopathy (mesenteric, para-aortic, or pelvic). 
Table 3. Variation in the proportion of biopsies performed by either CNB or EB among patients with lymphadenopathy between 2002 and 2017

\begin{tabular}{lcccc}
\hline Total & $\begin{array}{l}2002 \\
(n=33)\end{array}$ & $\begin{array}{l}2007 \\
(n=28)\end{array}$ & $\begin{array}{l}2012 \\
(n=44)\end{array}$ & $\begin{array}{l}2017 \\
(n=60)\end{array}$ \\
\hline $\mathrm{CNB}, n(\%)$ & $4(12)$ & $7(25)$ & $18(41)$ & $47(77)$ \\
$\mathrm{EB}, n(\%)$ & $29(88)$ & $21(75)$ & $26(59)$ & $13(23)$ \\
Days from the first visit to diagnosis, median (range) & $13.0(7.0,21.0)$ & $13.5(7.0,1853.0)$ & $15.0(6.0,192.0)$ \\
CNB & $14.0(12.0,17.5)$ & $21.5(7.0,271.0)$ & $22.0(12.0,80.0)$ \\
EB & $21.0(18.0,32.0)$ & $25.0(10.0,63.0)$ & 21.004 \\
\hline value & 0.0438 & 0.001 & 0.189 & 0.004 \\
\hline
\end{tabular}

The comparison of the time spent on diagnosing between CNB and EB during the same period. EB, excisional biopsy; CNB, core-needle biopsy.

$18 \%$, and 1 and 5 in 6\% each. Two patients experienced 7 and 10 passes each. In terms of diagnostic yield, only one out of 72 patients underwent biopsy with CNB and subsequent EB due to insufficiency. Despite unsuccessful classification in 4 low-grade lymphoma cases, they were treated based only on CNB results. Therefore, they were not counted as cases that lacked adequate tissue for diagnosis.

\section{Biopsy Procedure-Related Complications}

In 2017, overall, 2 (2.7\%) out of 72 patients who underwent CNB experienced minor bleeding, and 1 (7.1\%) out of 14 patients who underwent $\mathrm{EB}$ had minor bleeding. All these complications were graded as grade IIIa according to the Clavien-Dindo classification.

\section{Comparison between CNB and EB in Biopsy for Lymphadenopathy}

We also studied patients who underwent biopsy for truncal lymphadenopathy to compare the characteristics of CNB with those of EB. For this analysis, we focused on truncal lymphadenopathy including superficial lymphadenopathy (cervical, axillary, or inguinal) and abdominal lymphadenopathy (mesenteric, para-aortic, or pelvic), and anterior mediastinal lymphadenopathy to exclude potential bias related to biopsy sites. The results are summarized in Table 2.

Patient age was found to be comparable between the CNB group and the EB group (median 67 years old, range 26-89 and median 61 years old, range $18-80$ years, $p=$ $0.66)$. Irrespective of the subtypes of malignant lymphomas such as aggressive or indolent, the frequency of $\mathrm{CNB}$ and $\mathrm{EB}$ was consistent. However, $\mathrm{CNB}$ was chosen significantly more frequently for biopsy for deep lesions, which include anterior mediastinal mass and abdominal lymph node, than for biopsy for superficial lymph nodes (53 vs. $8 \%, p=0.004$ ). The size of the target lesion resected by CNB measured by imaging was larger than that resected by EB (median $4.2 \mathrm{~cm}$, range $1.0-15.0 \mathrm{~cm}$ and median $2.8 \mathrm{~cm}$, range $0.7-4.8 \mathrm{~cm}$, respectively, $p=0.002$ ).

In terms of diagnostic yield, all the biopsies with either EB or CNB resulted in actionable diagnosis (Table 2). The number of immunohistochemical slides required for an actionable diagnosis was almost the same in both the CNB group and the EB group (median 6, range 4-14 and median 7 , range $6-14$ respectively, $p=0.12$ ). Regarding DLBCL, the cell of origin was determined successfully in 17 out of 20 patients (17 in the CNB group and 3 in the EB group) in this cohort. On the contrary, ancillary examination including G-banding, FISH, and flow cytometry was performed less frequently in patients diagnosed with CNB. More precisely, ancillary tests were performed in only 4 out of 47 patients who underwent biopsy with CNB. One patient with follicular lymphoma and 3 with diffuse large B-cell lymphoma underwent FISH on formalin-fixed paraffin-embedded slides. However, flow cytometry and G-banding were performed in 10 and 7 out of 13 patients who underwent biopsy with EB, respectively. None underwent FISH examination in the EB group. The median time from the first outpatient visit to biopsy was significantly shorter with $\mathrm{CNB}$ than with $\mathrm{EB}$ (median 5.5 days, range $0-185$ and median 15 days, range $0-67$ days, $p=0.01$ ). The time required for the diagnosis from the first visit was also significantly shorter with CNB than with EB (median 15 days, range 6-192 and median 22 days, range $12-87$ days, $p=0.004$ ). During the 15 years, the time necessitated for the diagnosis from the first visit was consistently shorter in the CNB group than in the EB group, as shown in Table 3. 
Table 4. Summary of advantageous and disadvantageous respects in CNB and EB

\begin{tabular}{lll}
\hline Method & CNB & EB \\
\hline Advantages & $\begin{array}{l}\text { Less invasive procedure } \\
\text { The time required for the diagnosis from the first visit is } \\
\text { shorter }\end{array}$ & $\begin{array}{l}\text { Larger tissue volume (preferred for evaluation of histologic architecture, } \\
\text { additional studies including genetic study, and banking of tissue for } \\
\text { future research) }\end{array}$ \\
\hline Disadvantages & $\begin{array}{l}\text { Smaller tissue volume (limitation in evaluation of histologic } \\
\text { architecture, additional studies including genetic study, and } \\
\text { banking of tissue for future research) } \\
\text { Skilled radiologist is required }\end{array}$ & $\begin{array}{l}\text { More invasive } \\
\text { General anesthesia is required for patients with deep-seated lesion } \\
\text { Often difficulty in coordinating surgery appointment such as surgery slot } \\
\text { The time required for the diagnosis from the first visit is longer }\end{array}$ \\
\hline
\end{tabular}

CNB, core-needle biopsy; EB, excisional biopsy.

\section{Discussion}

In the present study, we have shown that the use of $\mathrm{CNB}$ has been increasing as the initial diagnostic approach during the past 15 years. As far as we know, this is the first report to describe trend in increasing use of $\mathrm{CNB}$ for lymphoma diagnosis, availability of pathological slides for immunostaining, and the use of ancillary diagnostic tests using samples obtained through $\mathrm{CNB}$ in a clinical practice setting.

Although the frequency of performing ancillary tests was lower in the CNB group than in the EB group, the number of slides used for immunohistochemical staining was comparable between the groups. Moreover, CNB aided in obtaining samples from representative and/or deep-seated lesions. Furthermore, CNB was able to shorten the time required to make an actionable diagnosis from the first visit by approximately 10 days. The advantages and disadvantages of $\mathrm{CNB}$ and $\mathrm{EB}$ as a biopsy method for lymphoma diagnosis are summarized in Table 4. Representative guidelines recommend EB or tissue resection to diagnose malignant lymphomas; however, several studies have revealed that $\mathrm{CNB}$ has been increasingly used as the primary diagnostic modality for malignant lymphomas. At our institute, the frequency of CNB has increased dramatically $>4$-fold over the past 15 years. Regarding safety, no serious complications related to $\mathrm{CNB}$ were observed in our study. Two out of 72 patients $(2.7 \%)$ experienced minor bleeding, which is consistent with the previously reported incidence of $0.14-7.5 \%$ [15-17]. Therefore, this study showed that CNB is a biopsy method that has minimal complication and less physical burden on patients.

To assess the feasibility of CNB to obtain sufficient specimens for an actionable diagnosis compared with that of EB, we showed the number of immunohistochem- ical slides in CNB was comparable with that in EB. Previous studies showed that the volume of tissue samples could correlate with the needle gauge and the number of passes obtained $[18,19]$. While there was no significant difference, CNB with larger core needles (19 gauge or larger) tend to obtain greater tissue volume, which increase the diagnostic accuracy [18]. At our institution, among patients who had biopsy for lymphadenopathy or anterior mediastinal mass, $>80 \%$ of CNB was performed with 18-gauge core needles and with at least $>3$ passes. Our findings support the concept that larger samples obtained using a larger core needle may be helpful to diagnose malignant lymphomas. However, in the ECOG/ ACRIN 1412, in which nearly half of all patients were diagnosed by $\mathrm{CNB}$, over $40 \%$ of all the rejected specimens were obtained by CNB because of insufficient material for diagnosis and/or necessary molecular/genetic examinations [20]. In the present study, there was only 1 patient with insufficient tissue obtained with CNB. It is certain that ancillary tests that require fresh samples, such as flow cytometry and conventional karyotyping, were performed less frequently for $\mathrm{CNB}$ than for $\mathrm{EB}$. With additional passes we could have done more ancillary tests. However, it was policy at our institution to limit the number of pass for CNB during the study period. Therefore, we consider that sample volume obtained by $\mathrm{CNB}$, at least with the procedure that we have performed, may be sufficient for an actionable diagnosis. But it is difficult to conclude tissue volume obtained by CNB is enough for ancillary tests.

In terms of clinical benefits, in this study, the time needed for an actionable diagnosis from the first visit in patients with $\mathrm{CNB}$ was approximately 10 days shorter than that in patients with EB. This is largely because CNB can be performed in the outpatient clinic or IR room. However, it is generally required to schedule the operat- 
ing room and staff in advance when planning an EB. Johl et al. [21] reported that the time needed for diagnosis was similar in the CNB group (mean 5.3 days) and the EB group (mean 6.0 days) based on a retrospective analysis in Germany; it was unclear whether they counted time from the first visit as calculated in our study or from biopsy. Moreover, in the present study, CNB was chosen more frequently in patients with deep-seated lesions than in patients with superficial lesions (Table 2). Probably, it reflects the fact that EB for deep-seated lesions requires open or laparoscopic surgery, which is a more invasive procedure and usually requires a longer time for waiting than CNB. For example, in Japan, the waiting time for elective abdominal surgery such as gastrectomy is reported to be approximately 30 days [22]. In addition, the nodal size that was biopsied with CNB was significantly larger than that biopsied with EB. CNB may aid in obtaining a larger and more representative lesion than EB. EB sometimes ends up with biopsy of smaller reactive lymph nodes around the lymphoma lesion because it is sometimes difficult to approach a large tumor.

There are several limitations to the present study. First, this was a retrospective study conducted in a single institution with highly experienced experts in IR and with limited availability of outpatient surgery. The availability of slots for $\mathrm{CNB}$ or EB that can be related to time to biopsy will be heavily influenced by facility-specific circumstances. Moreover, yields and risk of complications of CNB vary according to facility or physician. Second, as discussed earlier, whether CNB is enough for a diagnosis of lymphoma could not be assessed in the present study. $\mathrm{CNB}$ appears to have a limitation in evaluating the histological architecture and in achieving enough samples for ancillary testing such as molecular testing. Such limitations would be relative. For example, histological architecture can be evaluated, at least in part, with the assistance of immunostaining for CD21 and CD23, which we did not routinely use during the study period. Moreover, with more passes for CNB, we could obtain enough tissue to perform more ancillary testing. Last, selection of additional tests was clearly affected by the diagnostic policy at our institution during the study period. For example, we did not routinely perform flow cytometry in CNB samples mainly because of the small sample size.

In summary, CNB has become a more frequently used primary diagnostic modality for lymphoma diagnosis at our institution. Regarding pathological evaluation, the number of slides for immunohistochemical staining was comparable between the $\mathrm{CNB}$ group and the EB group. Moreover, CNB was significantly preferred for sampling from deep-seated lesions, and $\mathrm{CNB}$ was able to target larger lesions than EB. Last but not least, the required time from the first visit to a final diagnosis was significantly shorter in patients who underwent CNB than those who underwent EB. However, tissue volume with CNB sometimes may be insufficient for subtyping or additional ancillary examinations at least with the CNB procedure that we have performed. Therefore, EB is still a standardized biopsy method for lymphoma diagnosis, but CNB can be an alternative option in clinical scenarios where EB is difficult to perform.

\section{Statement of Ethics}

The present study was approved by the Ethics Committee of the National Cancer Center (Approval number: 2017-161). All procedures followed were in accordance with the ethical standards of the responsible committee and with the Helsinki Declaration. The need for written informed consent was waived by the Ethics Committee because of the retrospective nature of the study.

\section{Conflict of Interest Statement}

The authors declare the following competing interests: S.M. reports personal fees from Bristol-Myers Squibb, Celgene, Chugai, Daiichi-Sankyo, Eisai, Novartis, SymBio, and Takeda. S.F. reports personal fees from Chugai, Otsuka, Takeda, and Zenyaku kogyo. T.S. reports personal fees from Chugai. D.M. reports grants and personal fees from Bristol-Myers Squibb, Celgene, Chugai, Janssen, Ono, and Takeda; and reports grants from Amgen Astellas BioPharma, Astellas Pharma, Merck, Novartis Pharma, Otsuka, and Sanofi; and reports personal fees from Eisai, Kyowa Kirin, NIPPON SHINYAKU, Synmosa Biopharma, and Zenyaku Kogyo. K.I. reports receiving research support from MSD, AstraZeneca, Abbvie, Incyte, Celgene, Novartis, Janssen, Yakult, Daiichi Sankyo, Chugai, and Beigene; and reports personal fees from Chugai, Celgene, Takeda, Novartis, Abbvie, Janssen, Kyowa Kirin, Eisai, MSD, Astra Zeneca, FUJIFILM Toyama Chemical, and Ono.

\section{Funding Sources}

The authors did not receive any funding.

\section{Author Contributions}

K.I. and Y.I. performed the research, analyzed the data, and wrote the initial manuscript. A.M.M. and H.T. contributed to pathologic assessment. M.S. performed $\mathrm{CNB}$ and supervised the biopsy procedures. The remaining authors reviewed the manuscript and provided patient care. 


\section{References}

1 Cheson BD, Fisher RI, Barrington SF, Cavalli F, Schwartz LH, Zucca E, et al. Recommendations for initial evaluation, staging, and response assessment of hodgkin and non-hodgkin lymphoma: the lugano classification. J Clin Oncol. 2014 Sep;32(27):3059-68.

2 Swerdlow SH, Campo E, Harris N, Jaffe ES, Pileri SA, Stein H, et al. World Health organization classification of tumours of haematopoietic and lymphoid tissue. 4th ed. Lyon, France: IARC Press; 2017. Vol. 2.

3 Eichenauer DA, Aleman BMP, André M, Federico $M$, Hutchings $M$, Illidge $T$, et al. Hodgkin lymphoma: ESMO clinical practice guidelines for diagnosis, treatment and follow-up. Ann Oncol. 2018 Oct;29 Suppl 4(Suppl 4): iv19-29.

4 Tilly H, Gomes da Silva M, Vitolo U, Vitolo U, Jack A, Meignan M, et al. Diffuse large Bcell lymphoma (DLBCL): ESMO clinical practice guidelines for diagnosis, treatment and follow-up. Ann Oncol. 2015 Sep;26 Suppl 5(Suppl 5):v116-25.

5 Dreyling M, Ghielmini M, Rule S, Salles G, Vitolo U, Ladetto M. Newly diagnosed and relapsed follicular lymphoma: ESMO clinical practice guidelines for diagnosis, treatment and follow-up. Ann Oncol. 2016 Sep;27(Suppl 5):v83-90.

6 d'Amore F, Gaulard P, Trümper L, Corradini P, Kim WS, Specht L, et al. Peripheral T-cell lymphoma: ESMO clinical practice guidelines for diagnosis, treatment and follow-up. Ann Oncol. 2015 Sep;26(Suppl 5):v108-15.

7 National Comprehensive Cancer Network (NCCN). NCCN clinical practice guidelines in oncology (NCCN guidelines): Hodgkin lymphoma. Version 2.2019. [cited August 13, 2019] Available from: https://www.nccn.org/ professionals/physician_gls/pdf/hodgkins. pdf.
8 National Comprehensive Cancer Network $(\mathrm{NCCN})$. NCCN clinical practice guidelines in oncology (NCCN guidelines): B-cell lymphoma. Version 4.2019. [cited August 13, 2019] Available from: https://www.nccn.org/ professionals/physician_gls/pdf/b-cell.pdf.

9 National Comprehensive Cancer Network (NCCN). NCCN clinical practice guidelines in oncology (NCCN guidelines): T-cell lymphoma. Version 2.2019. [cited August 13, 2019] Available from: https://www.nccn.org/ professionals/physician_gls/pdf/t-cell.pdf.

10 Frederiksen JK, Sharma M, Casulo C, Burack WR. Systematic review of the effectiveness of fine-needle aspiration and/or core needle biopsy for subclassifying lymphoma. Arch Pathol Lab Med. 2015 Feb;139(2):245-51.

11 Seviar D, Yousuff M, Chia Z, Ramesar K, Newman J, Howlett DC. Image-guided core needle biopsy as the first-line diagnostic approach in lymphoproliferative disorders - a review of the current literature. Eur J Haematol. 2021 Feb;106(2):139.

12 Li J, Han J, Wang Y, Mo Y, Li J, Xiang J, et al. Core needle biopsy targeting the viable area of deep-sited dominant lesion verified by color doppler and/or contrast-enhanced ultrasound contribute to the actionable diagnosis of the patients suspicious of lymphoma. Front Oncol. 2020 Oct 7;10:500153.

13 Katayama H, Kurosawa Y, Nakamura K, Ito H, Kanemitsu Y, Masuda N, et al. Extended Clavien-Dindo classification of surgical complications: Japan clinical oncology group postoperative complications criteria. Surg Today. 2016 Jun;46(6):668-85.

14 Kanda Y. Investigation of the freely available easy-to-use software "EZR" for medical statistics. Bone Marrow Transplant. 2013 Mar; 48(3):452-8.
15 Pappa VI, Hussain HK, Reznek RH, Whelan J, Norton AJ, Wilson AM, et al. Role of imageguided core-needle biopsy in the management of patients with lymphoma. J Clin Oncol. 1996 Sep;14(9):2427-30.

16 de Kerviler E, Guermazi A, Zagdanski AM, Meignin V, Gossot D, Oksenhendler E, et al. Image-guided core-needle biopsy in patients with suspected or recurrent lymphomas. Cancer. 2000 Aug;89(3):647-52.

17 Han F, Xu M, Xie T, Wang JW, Lin QG, Guo ZX, et al. Efficacy of ultrasound-guided core needle biopsy in cervical lymphadenopathy: A retrospective study of 6,695 cases. Eur Radiol. 2018 May;28(5):1809-17.

$18 \mathrm{Hu}$ Q, Naushad H, Xie Q, Al-Howaidi I, Wang $\mathrm{M}, \mathrm{Fu}$ K. Needle-core biopsy in the pathologic diagnosis of malignant lymphoma showing high reproducibility among pathologists. Am J Clin Pathol. 2013 Aug;140(2):238-47.

19 Burke C, Thomas R, Inglis C, Baldwin A, Ramesar K, Grace R, et al. Ultrasound-guided core biopsy in the diagnosis of lymphoma of the head and neck. A 9 year experience. Br J Radiol. 2011 Aug;84(1004):727-32.

20 King RL, Nowakowski GS, Witzig TE, Scot DW, Little FR, Hong F, et al. Rapid, real time pathology review for ECOG/ACRIN 1412: a novel and successful paradigm for future lymphoma clinical trials in the precision medicine era. Blood Cancer J. 2018 Feb;8(3):27.

21 Johl A, Lengfelder E, Hiddemann W, Klapper W; German Low-grade Lymphoma Study Group. Core needle biopsies and surgical excision biopsies in the diagnosis of lymphomaexperience at the lymph node registry kiel. Ann Hematol. 2016 Aug;95(8):1281-6.

22 Kumazu Y, Oba K, Hayashi T, Yamada T, Hara K, Osakabe H, et al. Relationship between the waiting times for surgery and survival in patients with gastric cancer. World J Surg. 2020 Apr;44(4):1209-15. 